\title{
Velocity and turbulence at a wing-wall abutment
}

\author{
ABDUL KARIM BARBHUIYA and SUBHASISH DEY* \\ Department of Civil Engineering, Indian Institute of Technology, \\ Kharagpur 721 302, India \\ e-mail: sdey@civil.iitkgp.ernet.in
}

MS received 30 April 2003; revised 3 July 2003

\begin{abstract}
Experimental investigation of the 3D turbulent flow field around a $45^{\circ}$ wing-wall abutment, resting on a rough rigid bed, is reported. The experiment was conducted in a laboratory flume using the Acoustic Doppler Velocimeter (ADV). Profiles of time-averaged velocity components, turbulent intensity components, turbulent kinetic energy and Reynolds stresses at different azimuthal planes are presented. Vector plots of flow fields at azimuthal and horizontal planes show the presence of a primary vortex associated with the downflow in the upstream side of the abutment and a wake vortex on the downstream side. The shear stresses acting on the bed around the abutment are estimated from the Reynolds stresses and velocity gradients. The data presented in this study would be useful to researchers for future development and comparison of theoretical models of flow fields around bridge abutments.
\end{abstract}

Keywords. Abutments; three-dimensional flow; turbulent flow; open channel flow; hydraulics.

\section{Introduction}

When a wing-wall abutment is placed vertically on a rigid-bed rectangular channel by attaching it to one of the vertical sidewalls of the channel, the approaching turbulent boundary layer undergoes separation and rolles up to form the well-known primary vortex, which swept out by the side of the abutment. Limited research has been undertaken on the flow field around groynes and abutments (Rajaratnam \& Nwachukwu 1983; Ahmed \& Rajaratnam 2000). Kwan (1989) and Kwan \& Melville (1994) used the hydrogen bubble technique to measure the 3D flow field in a scour hole at a wing-wall abutment. However, the flow field (including turbulence characteristics) in the vicinity of a wing-wall abutment placed on a rough rigid bed still remains to be investigated.

The purpose of the present study is to investigate the 3D turbulent flow field in the vicinity of a wing-wall abutment, using the Acoustic Doppler Velocimeter (ADV). At different vertical planes, the time-averaged velocity components, turbulent intensity components, turbulent kinetic energy and Reynolds stresses were measured experimentally by the ADV. The flow

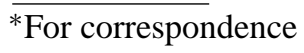


field around the abutment is represented using the measured velocity data through vector plots at different vertical and horizontal planes. The shear stresses on the bed are determined from the Reynolds stresses and velocity gradients.

\section{Experimental method and equipment}

The laboratory experiment was conducted in a flume of length $20 \mathrm{~m}$, width $0.9 \mathrm{~m}$ and depth $0.7 \mathrm{~m}$. A $45^{\circ}$ wing-wall abutment, made of transparent perspex, was attached to the glass sidewall of the flume resting on the flume bottom. The wing-wall abutment shown figure 1 has a streamwise length $b$ of $0.36 \mathrm{~m}$ and a transverse length $l$ of $0.12 \mathrm{~m}$. In order to get the fully developed turbulent flow at the test section, the abutment was placed at a distance of $12 \mathrm{~m}$ from the flume inlet. To simulate the turbulent flow over a rough planar sediment-bed, uniform sand having median diameter $d$ of $0.52 \mathrm{~mm}$ was glued over the flume bottom. Geometric standard deviation $\sigma_{g}$ of particle size distribution, given by $\left(d_{84} / d_{16}\right)^{0 \cdot 5}$, was $1 \cdot 21$, which was less than 1.4 for a uniformly graded sand (Dey et al 1995). However, the effect of smooth side-wall on flow field was negligible, as the width of the flume was $0.9 \mathrm{~m}$ being sufficient to reduce the side-wall effect. The flow discharge in the flume, controlled by an inlet valve, was measured by a calibrated V-notch weir fitted at the inlet of the flume. The flow depth, adjusted by a tailgate, was recorded by a Vernier point gage with an accuracy of $\pm 0.1 \mathrm{~mm}$. Initially, the flume was slowly filled with the water; and the measurement was started once the flow depth and the discharge reached to the desired values. The approaching flow depth $h$ was kept at $0.2 \mathrm{~m}$; and the experiment was run for an average approaching flow velocity $U$ of $0.294 \mathrm{~m} / \mathrm{s}$, which maintained a condition of $u * / u *_{c}=0.95$; where $u *$ is the approaching shear velocity; and $u *_{c}$ is the critical shear velocity of glued bed particles. The critical shear velocity $u *_{c}$ of glued bed particles was determined from the Shields diagram. The average approaching flow velocity $U$ was obtained from the measured vertical profile of approaching flow velocity at the mid-section of the flume, which was located at $2 \mathrm{~m}$ upstream of the center of the abutment, where the free flow occurred. The semi-logarithmic plot of the approaching flow velocity profile was used to determine approaching shear velocity $u *$.

A 5-cm downlooking acoustic Doppler velocimeter (ADV), developed by SonTek, was used to measure the instantaneous 3D velocity components. The ADV was operated on a pulseto-pulse coherent Doppler shift to provide 3D velocity components at a rate of $50 \mathrm{~Hz}$. The acoustic sensor consisted of one transmitting transducer and three receiving transducers. The receiving transducers were mounted on short arms the transmitting transducer at $120^{\circ}$ azimuth intervals. Acoustic beams with a frequency of $10 \mathrm{MHz}$ were emitted from the transmitting

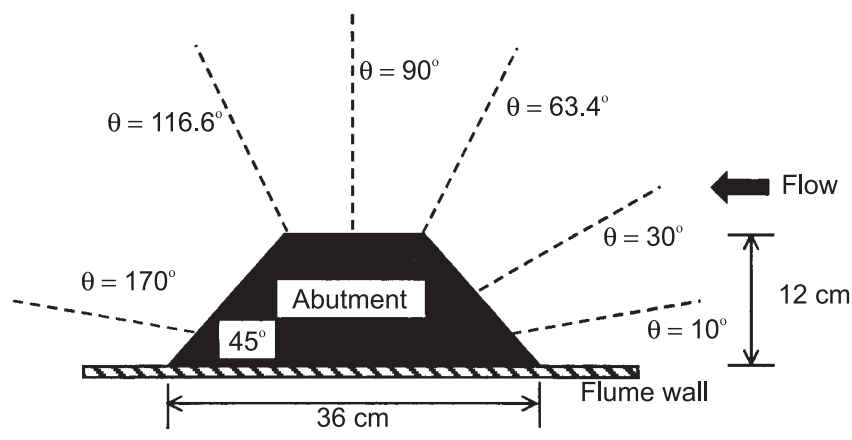

Figure 1. Sections of flow measurements. 
transducer. The beams traveling through the water arrived at the measuring point, located $5 \mathrm{~cm}$ below the transducer. They were reflected by the ambient particles within the flow being received by the receiving transducers. The processing module performed the digital signal processing required to measure the Doppler shifts. The data acquisition software provided real-time display of the data in graphical and tabular forms. There was no requirement of seeding of the flow during experiments, as the signal-noise ratio (SNR) was in the range of 12 to 16 . Because of the interference due to echoes from the flume bed, the receiving signal might be disturbed, which may result in inaccurate velocity measurement. In this study, a few points near the bed were found to be influenced in this way. The velocity measurement by the ADV probe was not possible in the zone, which was $5 \mathrm{~cm}$ below the free surface. The ADV readings were taken along several vertical lines in azimuthal planes of $\theta=10^{\circ}, 30^{\circ}, 63 \cdot 4^{\circ}, 90^{\circ}, 116 \cdot 6^{\circ}$ and $170^{\circ}$, as shown in figure 1 . Also, in order to get flow vectors at horizontal planes, the ADV readings were taken along different radial lines at horizontal planes of $z=0.05 \mathrm{~h}, 0.4 \mathrm{~h}$ and $0 \cdot 7 \mathrm{~h}$. Detailed description of experimental setup and procedure is given by Barbhuiya (2003).

\section{Velocity profiles}

The schematic diagram of a $45^{\circ}$ wing-wall abutment including cylindrical polar coordinate system is shown in figure 2 . It is convenient to use a cylindrical polar coordinate system due to the semicircular curvature of streamline pattern in the upstream of the abutment. The timeaveraged velocity components in $(\theta, r, z)$ are represented by $(u, v, w)$. At different azimuthal angles $\theta$, the velocity profiles are plotted in a nondimensional $\hat{r} \hat{z}$-plane; where $\hat{r}$ is $r / l$; and $\hat{z}$ is $z / l$. The flow field near the free surface is beyond the scope of this study, as the velocity readings were confined to the region $5 \mathrm{~cm}$ below the free surface due to the limitation of the ADV.

Figure 3 shows the vertical profiles of nondimensional time-averaged tangential velocity component $\hat{u}(=u / U)$ at different azimuthal planes. The tangential velocity $u$ represents the characteristics of the passage of approaching flow in the upstream and by the side of the abutment as a skewed velocity profile. At $\theta=10^{\circ}$, the magnitude of $u$ is relatively small as it originates beyond $0^{\circ}$ and grows with increase in azimuthal angle $\theta$. Hence, $u$ is zero at $0^{\circ}$.

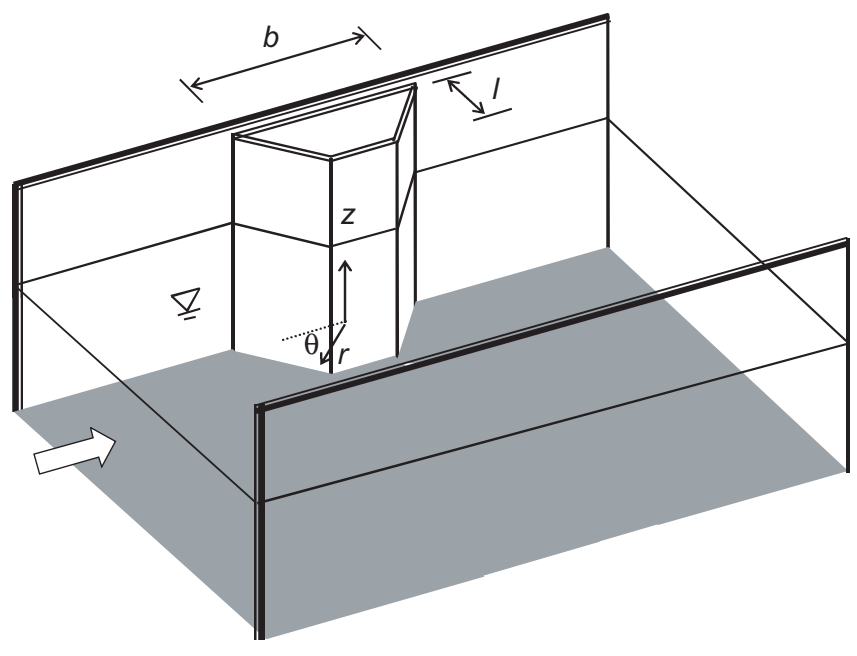

Figure 2. Schematic diagram of a $45^{\circ}$ wing-wall abutment and coordinate system. 

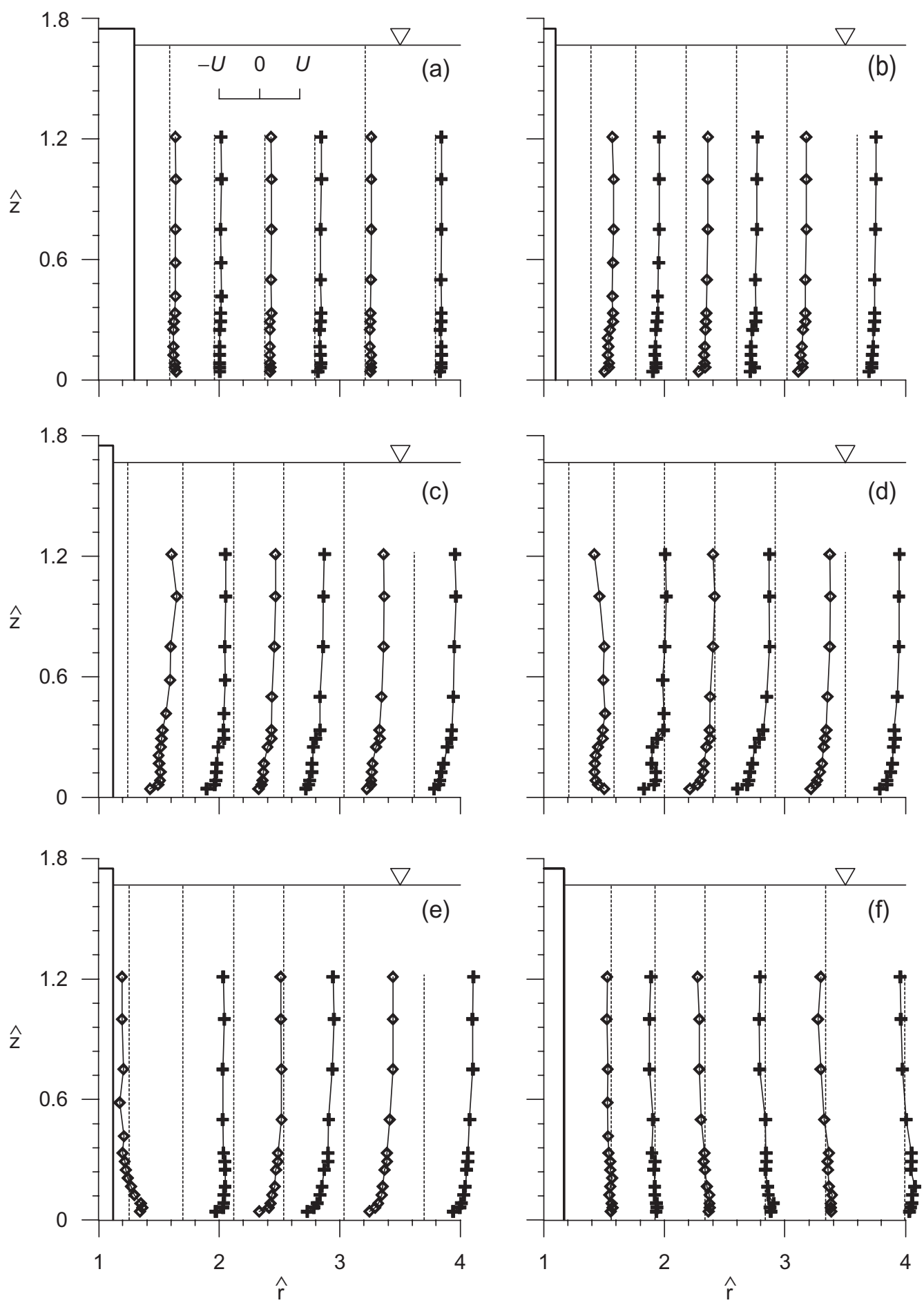

Figure 3. Vertical profiles of $\hat{u}$ at azimuthal sections $\theta=10^{\circ}(\mathbf{a}), 30^{\circ}(\mathbf{b}), 63.4^{\circ}(\mathbf{c}), 90^{\circ}(\mathbf{d}), 116 \cdot 6^{\circ}(\mathbf{e})$ and $170^{\circ}$ (f). 
The profiles of $u$ have a similarity with the turbulent flow profile over a planar bed and can, therefore, be represented by a power law in the upstream $\left(10^{\circ}-90^{\circ}\right)$ of the abutment. The passage of downflow by the side of the abutment increases $u$ considerably near the abutment at $63.4^{\circ}$ and $90^{\circ}$. Though no radial profile of $u$ is plotted, from the observation of the vertical profiles of $u$, it is evident that there is a pronounced skewness in the profile patterns. This is believed to be due to the effect of the primary vortex and its passage in the form of a helicoidal flow by the side of the abutment. The existence of helicoidal flow decreases $u$ to some extent, which is particularly apparent from the velocity profiles close to the bed. The primary vortex, which originates at the upstream of the abutment, is swept out by the tangential velocity $u$ by the side of the abutment. The magnitude of $u$ increases with increase in $\theta$ becoming a maximum at $63.4^{\circ}$. In the downstream, the tangential velocity diminishes to a great extent with further increase in $\theta$ beyond $116 \cdot 6^{\circ}$. At $170^{\circ}$, it shows a feeble $u$ as a result of wake due to the flow separation. The maximum magnitude of tangential velocity $u$ measured was $1.3 U$ at $\theta=63.4^{\circ}, r=1.25 l$ and $z=l$.

The vertical profiles of nondimensional time-averaged radial velocity component $\hat{v}(=$ $v / U)$ at different azimuthal planes are presented in figure 4 . At the upstream of the abutment $\left(10^{\circ}-90^{\circ}\right)$, it is evident from the radial velocity $v$ profile that the flow separation at the upstream of the abutment base produces a reversal nature of $v$ near the bed (it is distinct at $63 \cdot 4^{\circ}$ ). As the ADV reading is possible from $5 \mathrm{~mm}$ above the bed, the reversal nature is not very prominent. However, an extension of the existing velocity profiles at the bed confirms the reversal nature of flow near the abutment. Thus, $v$ changes sign near the bed close to the abutment, producing a helicoidal flow at the upstream of the abutment base. This may be compared with the radial velocity at a circular cylinder (Graf \& Yulistiyanto 1999). Close to the bed, the vertical profile of $v$ changes rapidly. But immediately above the bed, the variation of $v$ along $z$ being negative is almost logarithmic due to the effect of the approaching flow, becoming a maximum near the free surface. However, $v$ remains essentially almost logarithmic and progressively diminishes over the entire flow depth approaching towards the abutment. Although the radial profile of $v$ is not given, a close examination of the vertical profiles of $v$ reveals a nonlinear (approximately parabolic) variation of $v$ along $r$. Magnitude of $v$ decreases with decrease in $r$ due to the abutment boundary. Also, decreasing nature of $v$ with increase in $\theta$ for $10^{\circ}-90^{\circ}$ is evident as a result of primary vortex attenuation. The radial velocity $v$ becomes positive having no circulatory motion with further increase in $\theta$ beyond $90^{\circ}$. At $170^{\circ}$, it shows a flow reversal (towards the abutment) adjacent to the abutment as a result of backflow due to the flow separation. Further downstream, the flow reversal diminishes and the velocity profiles show a recovery being a part of the main flow away from the abutment.

Figure 5 exhibits the vertical profiles of nondimensional time-averaged vertical velocity component $\hat{w}(=w / U)$ at different azimuthal planes. The overall magnitude of vertical velocity $w$ is relatively weak. The characteristics of flow separation and resulting helicoidal flow are not very distinct due to smallness in magnitude of $w$. The variation of $w$ is nonlinear with negative value near the upstream face of the abutment. The negative value of $w$ in front of the abutment confirms that there is a downflow in that zone. The magnitude of $w$ remains negligible in approaching region $(r \geq l)$, but subsequently grows up towards the abutment. However, in the upstream $\left(10^{\circ}-90^{\circ}\right)$, the downflow that increases towards the abutment becomes a maximum close to the abutment. The maximum magnitude of $w$ measured was $0.25 U$ at $\theta=63.4^{\circ}$ and $z=0.3 l$. To be more explicit, at the upstream face of the abutment an adverse pressure gradient creates a downflow being returned at the base of the abutment, forming a counter-clockwise vortex (not distinct in this diagram), referred 

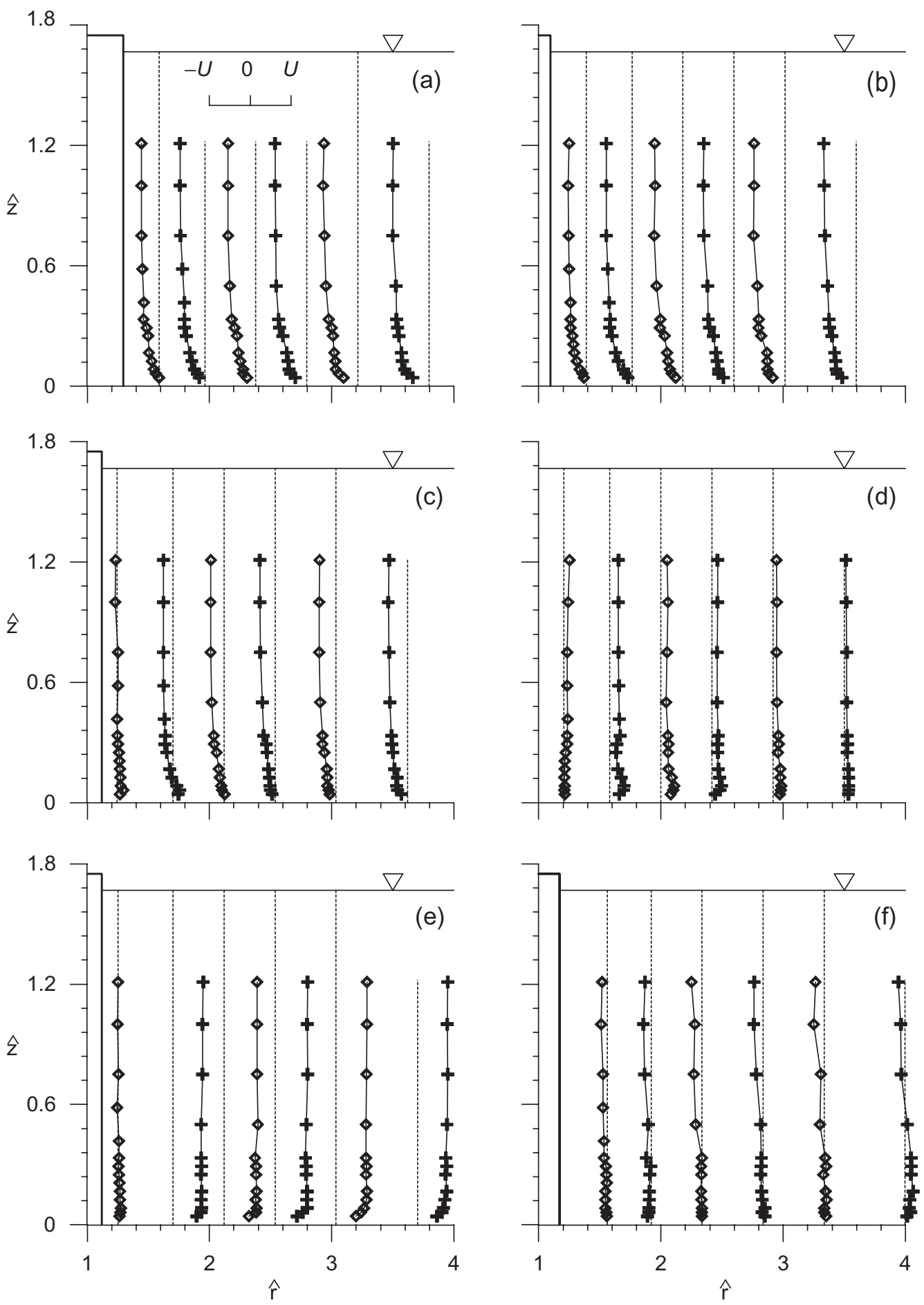

Figure 4. Vertical profiles of $\hat{v}$ at azimuthal sections $\theta=10^{\circ}(\mathbf{a}), 30^{\circ}(\mathbf{b}), 63 \cdot 4^{\circ}(\mathbf{c}), 90^{\circ}(\mathbf{d}), 116 \cdot 6^{\circ}(\mathbf{e})$ and $170^{\circ}$ (f). 
Velocity and turbulence at a wing-wall abutment
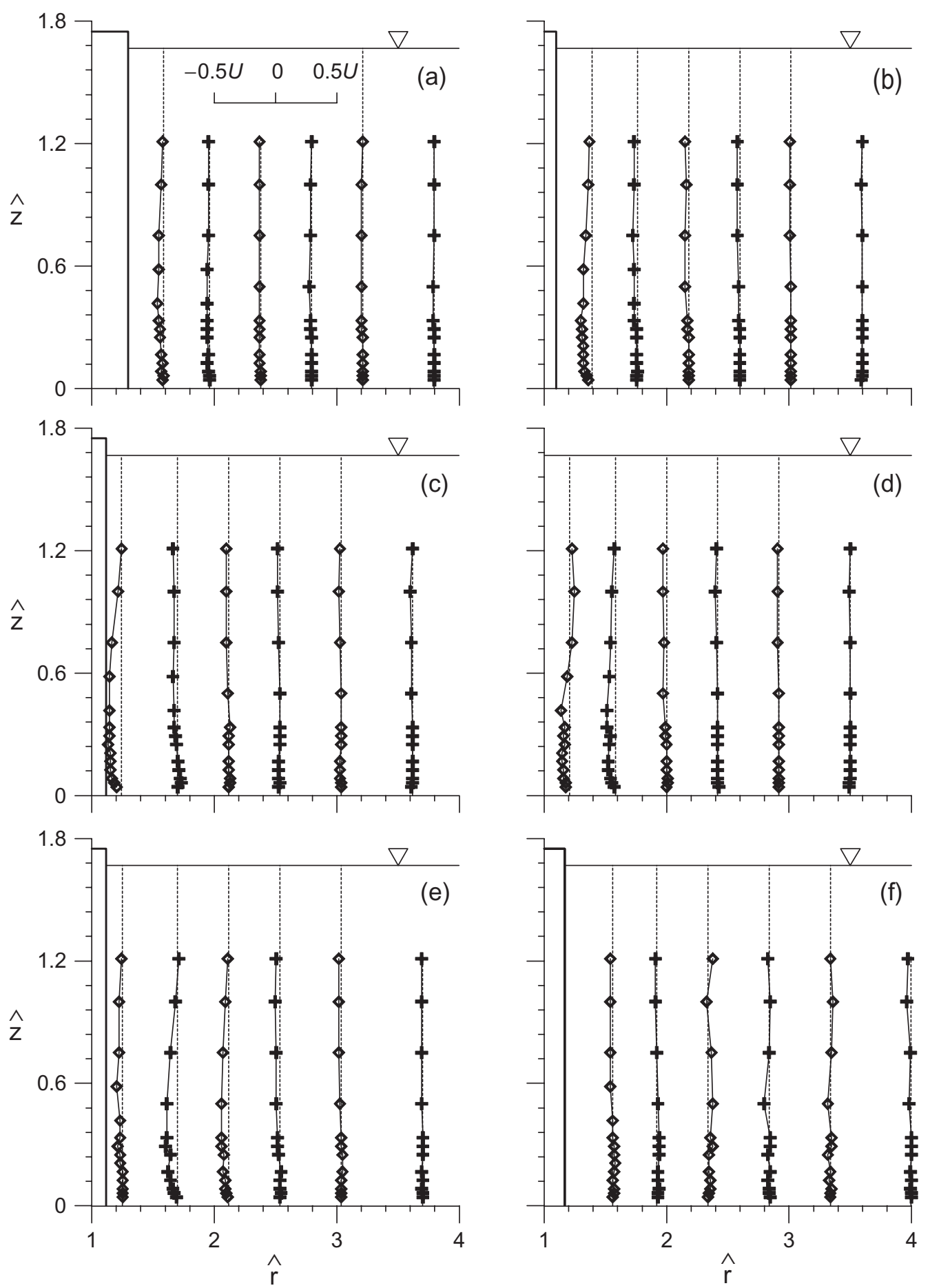

Figure 5. Vertical profiles of $\hat{w}$ at azimuthal sections $\theta=10^{\circ}(\mathbf{a}), 30^{\circ}(\mathbf{b}), 63 \cdot 4^{\circ}(\mathbf{c}), 90^{\circ}(\mathbf{d}), 116 \cdot 6^{\circ}(\mathbf{e})$ and $170^{\circ}$ (f). 
to as primary vortex. The magnitude of $w$ decreases with increase in $z$. As the magnitude of $w$ decreases with increase in $\theta$, the primary vortex attenuates with increase in $\theta$. In the downstream, at $170^{\circ}$, it shows a feeble upward flow; and further downstream, $w$ diminishes with the distance from the abutment.

The nondimensional time-averaged velocity vectors, whose magnitude and direction are $\left(\hat{v}^{2}+\hat{w}^{2}\right)^{1 / 2}$ and $\arctan (\hat{w} / \hat{v})$, respectively, at different azimuthal planes are shown in figure 6 .
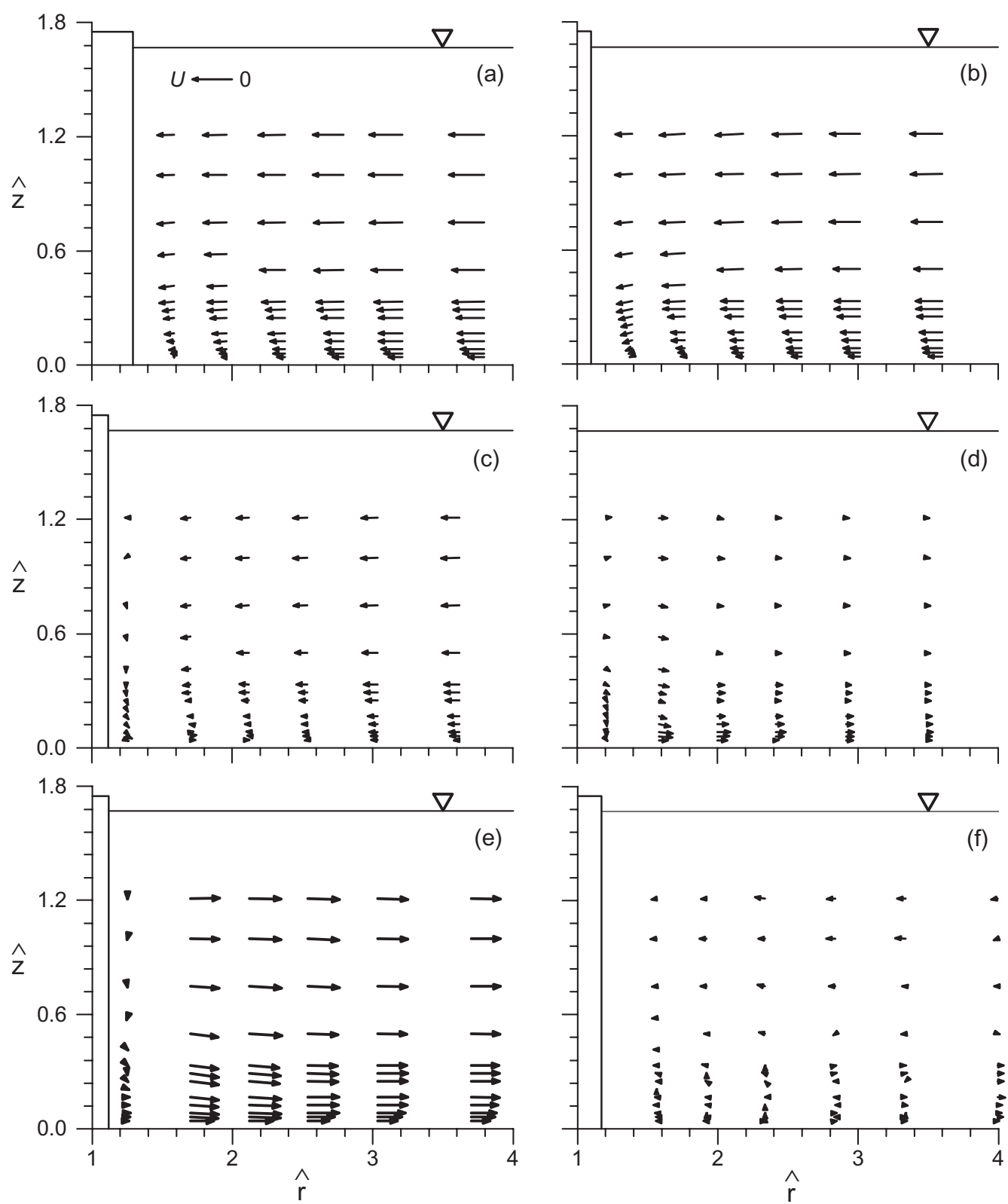

Figure 6. Nondimensional velocity vectors at azimuthal sections $\theta=10^{\circ}$ (a), $30^{\circ}$ (b), $63.4^{\circ}$ (c), $90^{\circ}(\mathbf{d}), 116 \cdot 6^{\circ}(\mathbf{e})$ and $170^{\circ}(\mathbf{f})$. 
The characteristics of the circulatory flow together with the downflow along the upstream face of the abutment are displayed. At the upstream, the flow is directed towards the abutment with diminishing magnitude. The circulation is strong at $10^{\circ}-63.4^{\circ}$ and decreases with increase in $\theta$. Above $z=0 \cdot 6$, the flow is almost horizontal, and below $z=0 \cdot 6$, the flow curves down near the abutment. However, the circulatory motion becomes very weak at $90^{\circ}$. In the downstream, at $116.6^{\circ}$, the flow directed away from the abutment is separated. On the other hand, at $170^{\circ}$, flow is reversed due to flow separation forming wake vortices. The nondimensional time-averaged velocity vectors, whose magnitude and direction are $\left(\hat{u}^{2}+\hat{v}^{2}\right)^{1 / 2}$ and arctan $(\hat{v} / \hat{u})$, respectively, at different horizontal planes are presented in figure 7 . The velocity vectors at different horizontal sections show the incoming motion of the approaching flow in front of the abutment having a smooth passage by the side of the abutment with a backflow adjacent to the abutment in the downstream. The backflow is distinct in the upper portion,

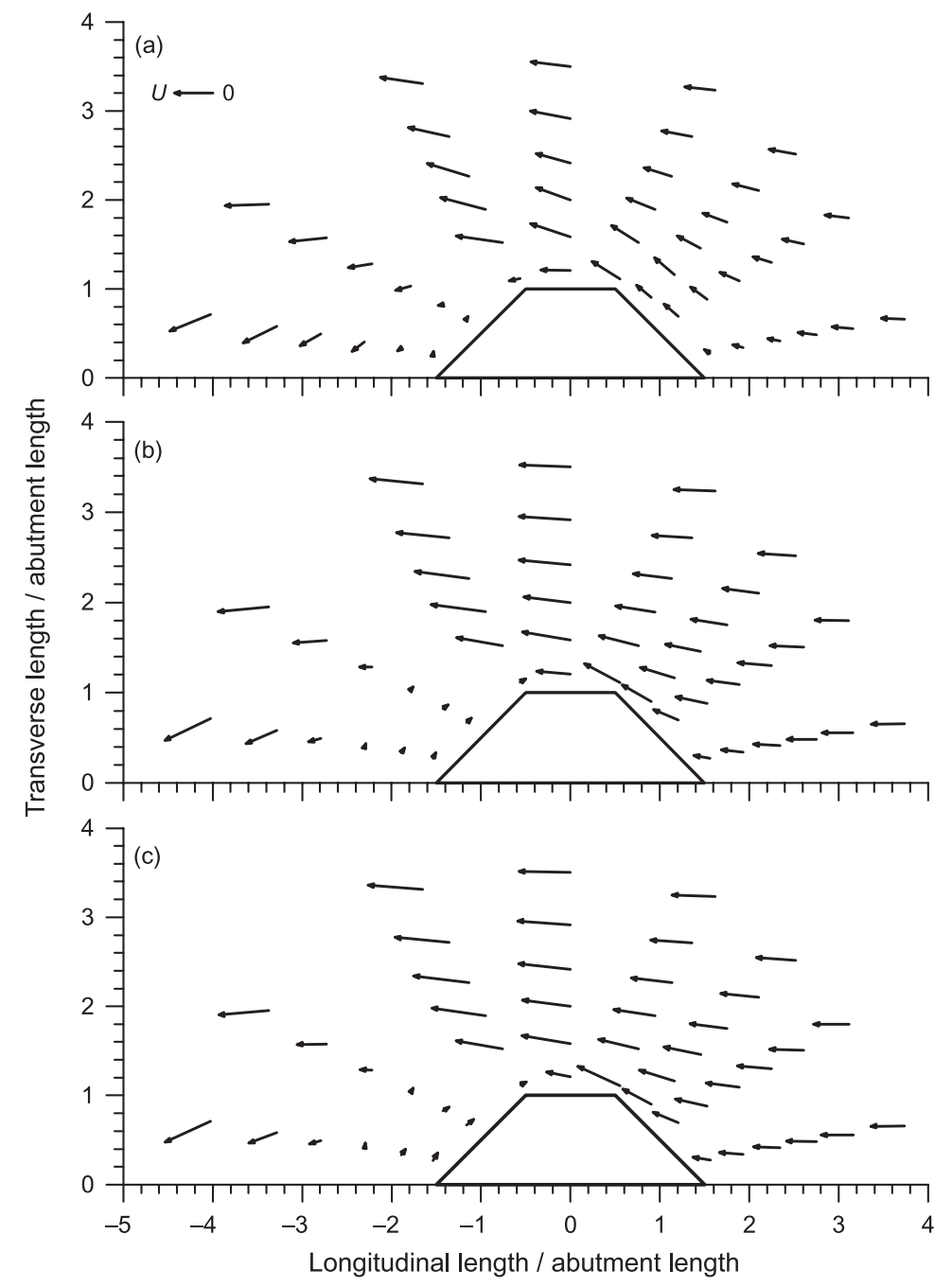

Figure 7. Nondimensional velocity vectors at sections $z=0.05$ (a), 0.4 (b) and $0.7 \mathrm{~h}(\mathbf{c})$. 
whereas the deflection in front of the abutment is more prominent in the lower portion. The nondimensional time-averaged absolute velocity $\hat{V}=\left(\hat{u}^{2}+\hat{v}^{2}+\hat{w}^{2}\right)^{1 / 2}$ contours at different azimuthal sections are depicted in figure 8 . At $10^{\circ}$ and $30^{\circ}$, the absolute velocity is circulatory as already shown in figures $6 \mathrm{a}$ and $\mathrm{b}$. On the other hand, at $90^{\circ}$, tangential velocity $u$ dominates
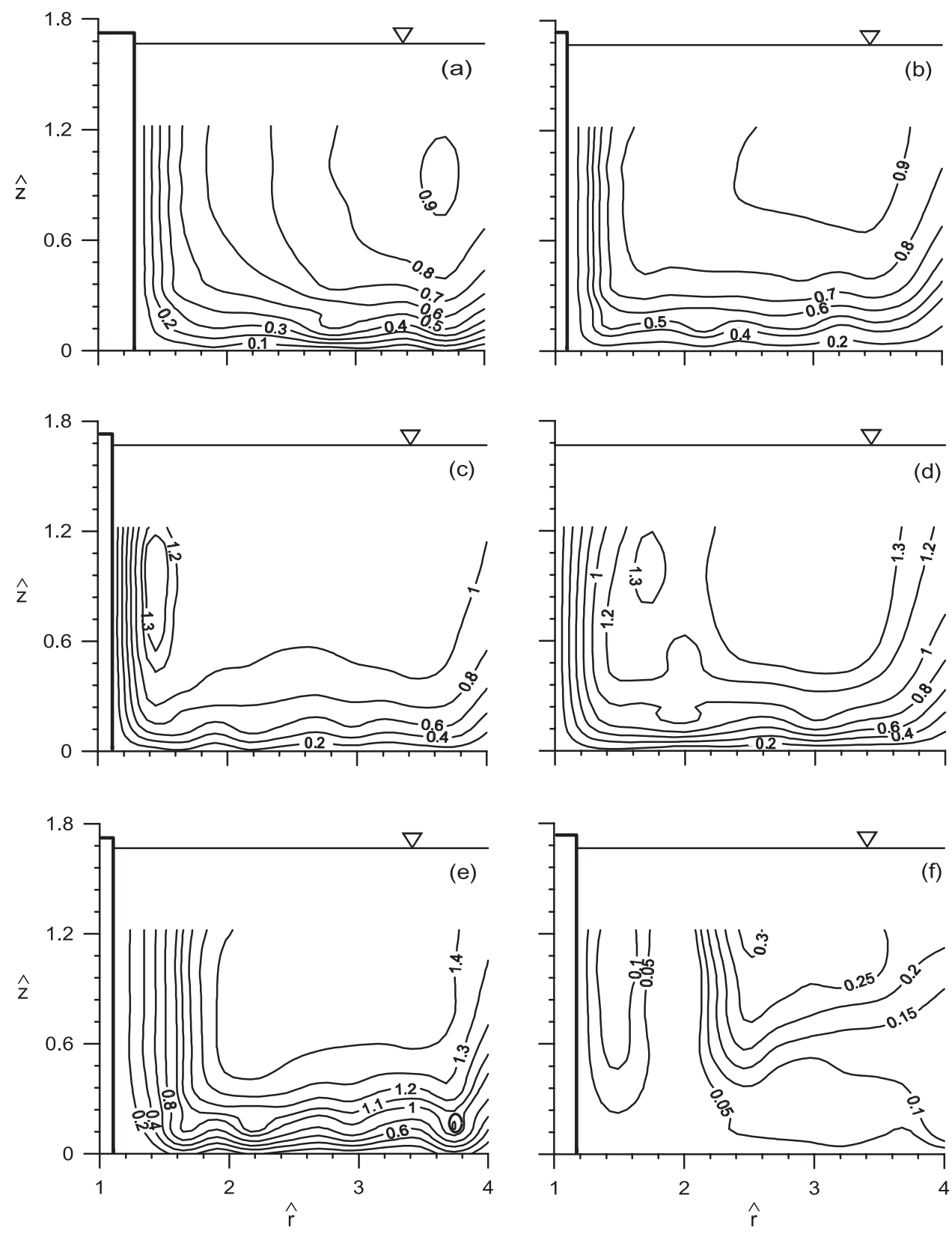

Figure 8. Nondimensional absolute velocity $\hat{V}$ contours at azimuthal sections $\theta=10^{\circ}$ (a), $30^{\circ}$ (b), $63 \cdot 4^{\circ}(\mathbf{c}), 90^{\circ}(\mathbf{d}), 116 \cdot 6^{\circ}(\mathbf{e})$ and $170^{\circ}(\mathbf{f})$. 
and the circulatory flow becomes weak. In other azimuthal planes, the diminishing nature of circulatory flow with increase in $\theta$ is displayed. At $63.4^{\circ}$ and $90^{\circ}$, the concentration of contour lines near the abutment refers to the region of rapid change of velocity magnitudes. However, gradual increase of velocity magnitude away from the abutment is observed at $116 \cdot 6^{\circ}$. The velocity contours in the downstream of the abutment show the flow becomes a part of the main flow away from the abutment.

From the preceding observations, the physics of the flow can be analyzed. The characteristic feature of the flow at a wing-wall abutment is a primary vortex flow along with skewed velocity profiles. The approaching flow separates upstream of the abutment, which is a concentric circular line known to be a line of separation, forming a vortex flow near the abutment. The downflow, developed due to the downward stagnation pressure gradient of the nonlinear approaching flow velocity along the adjacent vertical face of the abutment upstream, is pushed up by the vortex. The process of flow separation can be described using the concept of limiting streamline (Dey 1995). The limiting streamlines of the approaching flow along the original bed upstream and the reversed flow along the bed merge at the upstream of the abutment forming separated streamline. Thus, a surface of separation is produced in the form of an envelope. In this process, the approaching flow curves down towards the bed and rolls to form a primary vortex, which migrates downstream by the side of the abutment. The migration of the vortex flow along the circumference of the abutment towards the downstream may be compared with the passage of helicoidal flow in a channel bend (Rozovskii 1961). However, unlike the flow in a channel bend, the vertical velocity in the present case is substantially high due to a strong $3 \mathrm{D}$ vortex flow associated with a downflow close to the abutment. Again, the approaching flow deflects under the influence of the lateral pressure gradient created by the abutment. The slower-moving water near the abutment wall and the bed with less momentum turns more than the faster-moving water away from the abutment and the bed. Thus, the direction as well as the magnitude of the approaching velocity vector changes with the distance from the abutment wall and the bed causing the skewing nature of velocity profiles. In the downstream, the flow is ill defined and reversed due to the flow separation at the abutment side, which produces a wake downstream of it.

\section{Turbulent intensity}

The vertical profiles of nondimensional tangential turbulent intensity component $u^{+}[=$ $\left(\overline{u^{\prime 2}}\right)^{0 \cdot 5} / U$, where $u^{\prime}$ is the fluctuation of $u$ ] at different azimuthal planes are plotted in figure 9 . The tangential turbulent intensity remains almost unchanged above $z=0 \cdot 4$, having a profile more or less linear. Below $z=0 \cdot 4$, the flow is reversed near the bed. Therefore, $u^{+}$increases towards the bed. A most notable feature of the profile is the pronounced swells just below $z=0 \cdot 4$, owing to the flow separation. It is also evident that $u^{+}$remains almost unchanged with increase in $\theta$ up to $90^{\circ}$, but near the abutment $u^{+}$increases. Then the flow separates from the edge of the abutment, forming the wake in the downstream, where $u^{+}$increases considerably. But, the maximum intensity of $u^{+}$occurred near the outer vertical edges of the abutment. The shuddering effect of the primary vortex and the shedding of the wake vortices result in spikes near the bed in the upstream and downstream of the abutment. The vertical profiles of nondimensional radial turbulent intensity component $v^{+}\left[=\left(\overline{v^{\prime 2}}\right)^{0 \cdot 5} / U\right.$, where $v^{\prime}$ is the fluctuation of $v]$ and vertical turbulent intensity component $w^{+}\left[=\left(\overline{w^{\prime 2}}\right)^{0.5} / U\right.$, where $w^{\prime}$ is the fluctuation of $w$ ] at different azimuthal planes are presented in figures 10 and 11, respectively. The characteristics of the profiles of $v^{+}$are almost similar as that of $u^{+}$. 

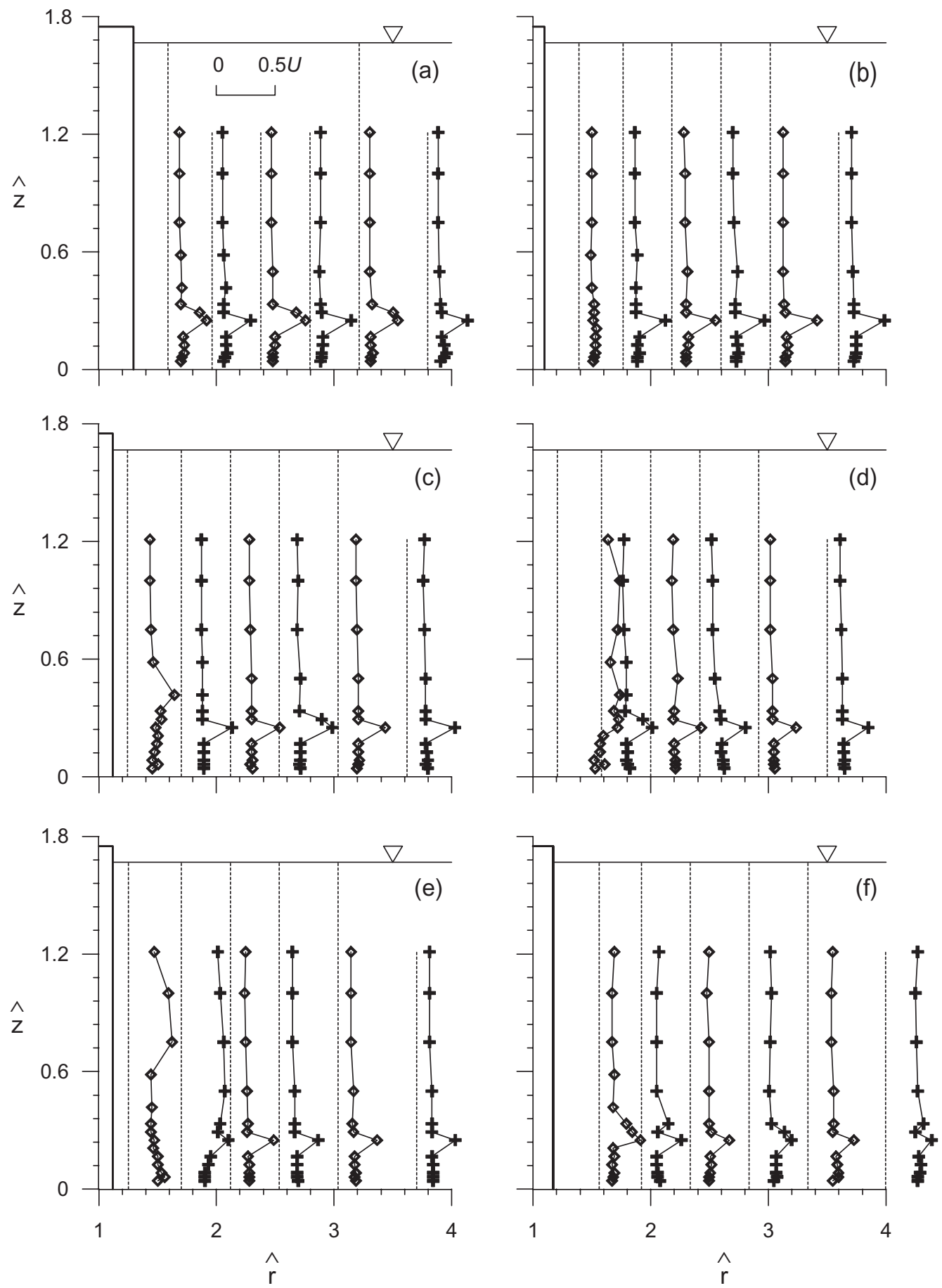

Figure 9. Vertical profiles of $u^{+}$at azimuthal sections $\theta=10^{\circ}$ (a), $30^{\circ}$ (b), $63.4^{\circ}$ (c), $90^{\circ}$ (d), $116 \cdot 6^{\circ}(\mathbf{e})$ and $170^{\circ}(\mathbf{f})$. 

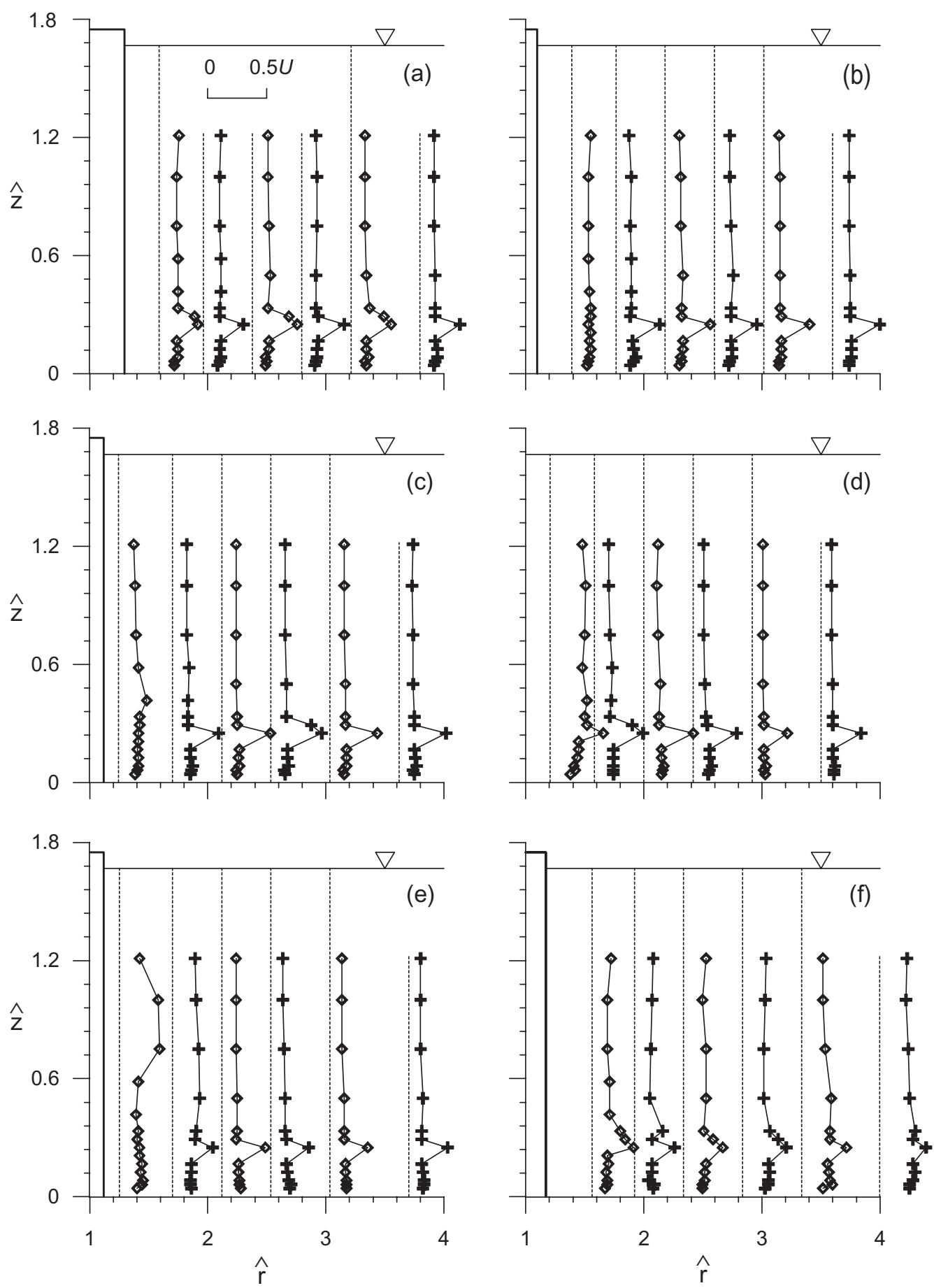

Figure 10. Vertical profiles of $v^{+}$at azimuthal sections $\theta=10^{\circ}(\mathbf{a}), 30^{\circ}(\mathbf{b}), 63.4^{\circ}(\mathbf{c}), 90^{\circ}(\mathbf{d})$, $116 \cdot 6^{\circ}(\mathbf{e})$ and $170^{\circ}(\mathbf{f})$. 

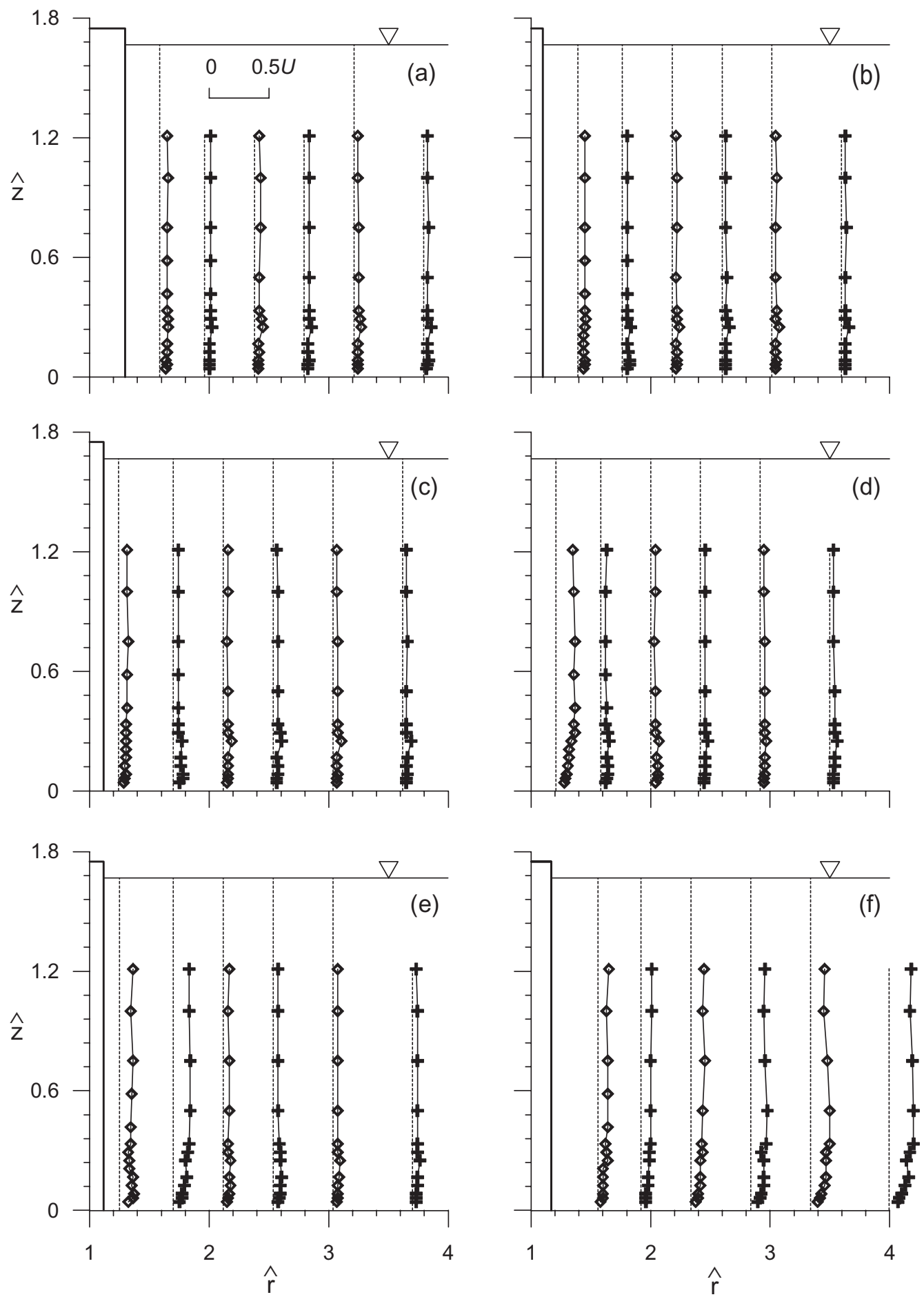

Figure 11. Vertical profiles of $w^{+}$at azimuthal sections $\theta=10^{\circ}(\mathbf{a}), 30^{\circ}(\mathbf{b}), 63.4^{\circ}(\mathbf{c}), 90^{\circ}$ (d), $116 \cdot 6^{\circ}(\mathbf{e})$ and $170^{\circ}$ (f). 
However, it is apparent that $w^{+}$are relatively less dominant ones and do not show any spike near the bed, because there is no shuddering effect or shedding of primary or wake vortex in the vertical direction. The vertical profiles of nondimensional turbulent kinetic energy $\hat{k}\left[=k / U^{2}=0 \cdot 5\left(u^{+2}+v^{+2}+w^{+2}\right)\right.$, where $k$ is the turbulent kinetic energy $]$ at different azimuthal planes, shown in figure 12, represent the cumulative effect of $u^{+}, v^{+}$and $w^{+}$. The swells result from the flow separation near the bed, and the kinetic energy becomes very strong over the entire depth behind and near the abutment. However, in the wake region, the ADV measurements do not have a high enough resolution to detect chaotic flow associated with the shedding of wake vortices. This is one of the possible causes of spikes in the profiles.

\section{Reynolds stresses}

Figures 13-15 display the vertical profiles of nondimensional Reynolds stresses $u w^{+}(=$ $\left.-\overline{u^{\prime} w^{\prime}} / u *^{2}\right), v w^{+}\left(=-\overline{v^{\prime} w^{\prime}} / u *^{2}\right)$ and $u v^{+}\left(=-\overline{u^{\prime} v^{\prime}} / u *^{2}\right)$ at different azimuthal planes. In the upstream, the Reynolds stresses at $10^{\circ}$ and $30^{\circ}$ are insignificant. For other azimuthal angles, $u w^{+}$and $v w^{+}$grow near the abutment. On the other hand, $u v^{+}$is relatively strong indicating more fluctuations in the horizontal plane. In the downstream, $u w^{+}, v w^{+}$and $u v^{+}$become considerably large with an inconsistent profile (inconclusive trend) due to the separation of flow and vortex shedding. However, $u v^{+}$changes signs near the abutment at $63 \cdot 4^{\circ}-90^{\circ}$.

\section{Bed shear stress}

The shear stress $\tau_{b}$ acting on the bed is estimated using the Reynolds stresses as follows:

$$
\tau_{b}=\left.\left(\tau_{\theta}^{2}+\tau_{r}^{2}\right)^{1 / 2}\right|_{\text {at bed }}
$$

where $\tau_{\theta}=-\rho\left(\overline{w^{\prime} u^{\prime}}+\overline{v^{\prime} u^{\prime}}\right), \tau_{r}=-\rho\left(\overline{u^{\prime} v^{\prime}}+\overline{w^{\prime} v^{\prime}}\right)$, and $\rho=$ mass density of water. The nondimensional bed shear stress $\hat{\tau}_{b}$ can be expressed as

$$
\tau_{b}=\frac{\tau_{b}}{\tau_{o}}=\left.\frac{1}{\tau_{o}}\left(\tau_{\theta}^{2}+\tau_{r}^{2}\right)\right|_{\text {at bed }}
$$

where $\tau_{o}=$ bed shear stress of approaching flow, that is $\rho u *^{2}$. The bed shear stress is also calculated from the velocity gradients, as was done by Melville \& Raudikivi (1977). The equation of nondimensional bed shear stress is

$$
\hat{\tau}_{b}=\frac{\rho(v+\varepsilon)}{\tau_{o}} \frac{\mathrm{d} q}{\mathrm{~d} z}
$$

where $\tau_{o}=$ kinematic viscosity of water (assumed as $10^{-6} \mathrm{~m}^{2} / \mathrm{s}$ ), $\varepsilon=$ eddy viscosity, and $q=$ velocity parallel to the bed at a normal distance $\mathrm{d} z(=5 \mathrm{~mm})$ above the bed, that is $\left(u^{2}+v^{2}\right)^{0.5}$ at $z=5 \mathrm{~mm}$. Since the eddy viscosity $\varepsilon$ is a function of circulatory flow (Odgaard 1986), it is a variable quantity around the abutment for different azimuthal angles $\theta$. Therefore, the values of $\hat{\tau}_{b}$ obtained from (2) is used to evaluate $\varepsilon$ using (3). As the values of $\varepsilon$ at different locations of an azimuthal angle $\theta$ are close, it is desirable to use an average value of $\varepsilon$ for an angle $\theta$. The average values of $\varepsilon$ estimated for different $\theta$ are given in table 1 . Figure 16 shows the variations of nondimensional bed shear stress $\hat{\tau}_{b}$ with $\hat{r}$ for different $\theta$ obtained using (2) 

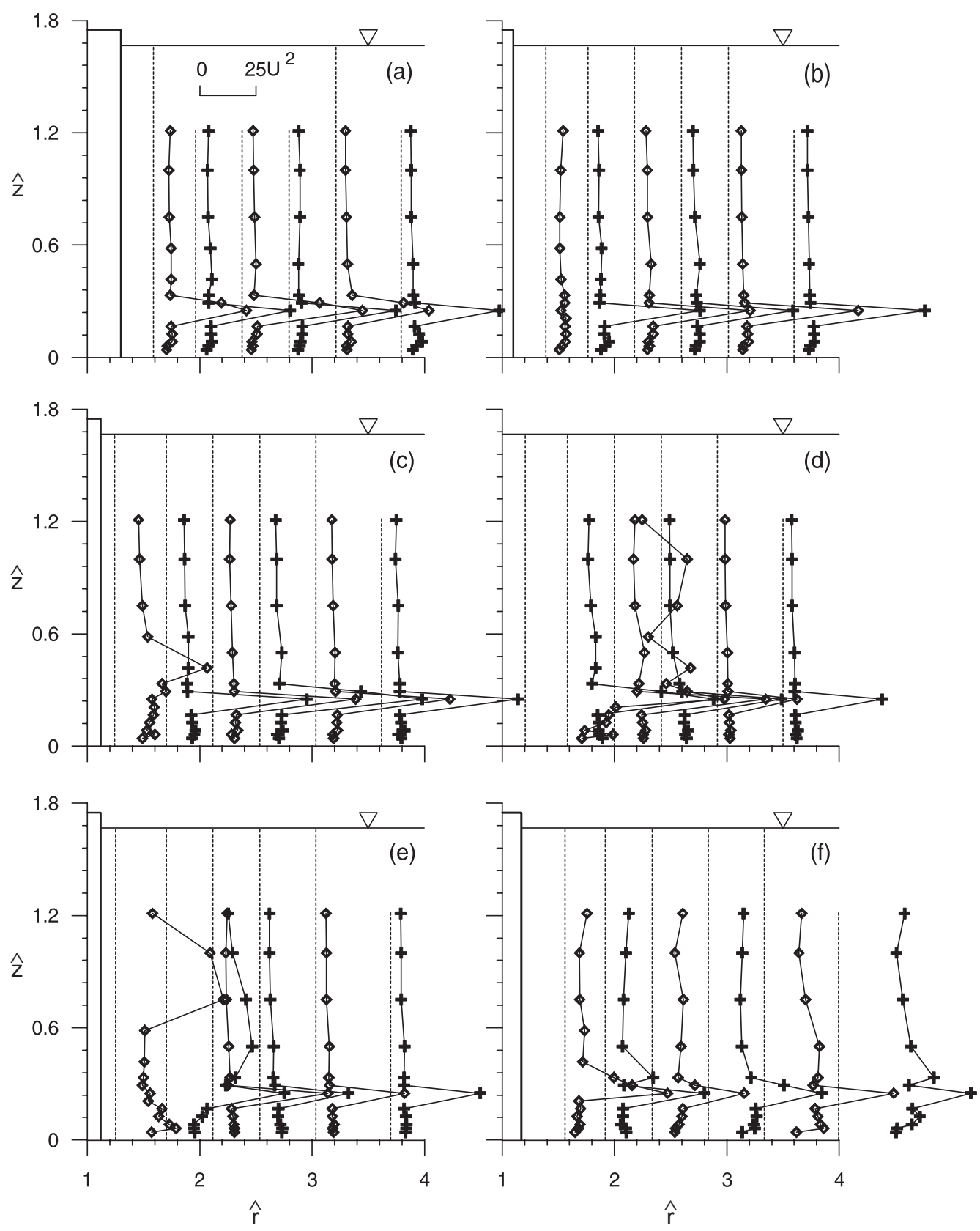

Figure 12. Vertical profiles of $\hat{k}$ at azimuthal sections $\theta=10^{\circ}$ (a), $30^{\circ}$ (b), $63.4^{\circ}$ (c), $90^{\circ}$ (d), $116 \cdot 6^{\circ}(\mathbf{e})$ and $170^{\circ}(\mathbf{f})$.

and (3). The bed shear stress increases with increase in $\theta$ significantly in the downstream. However, in most of the region, $\hat{\tau}_{b}$ is greater than unity except at $\theta=10^{\circ}$ and $30^{\circ}$. The shear stress is a maximum in the downstream. A similar result was also obtained by Melville \& Raudikivi (1977) for the flow around a cylindrical pier placed on flat bed (unscoured bed). 
Velocity and turbulence at a wing-wall abutment
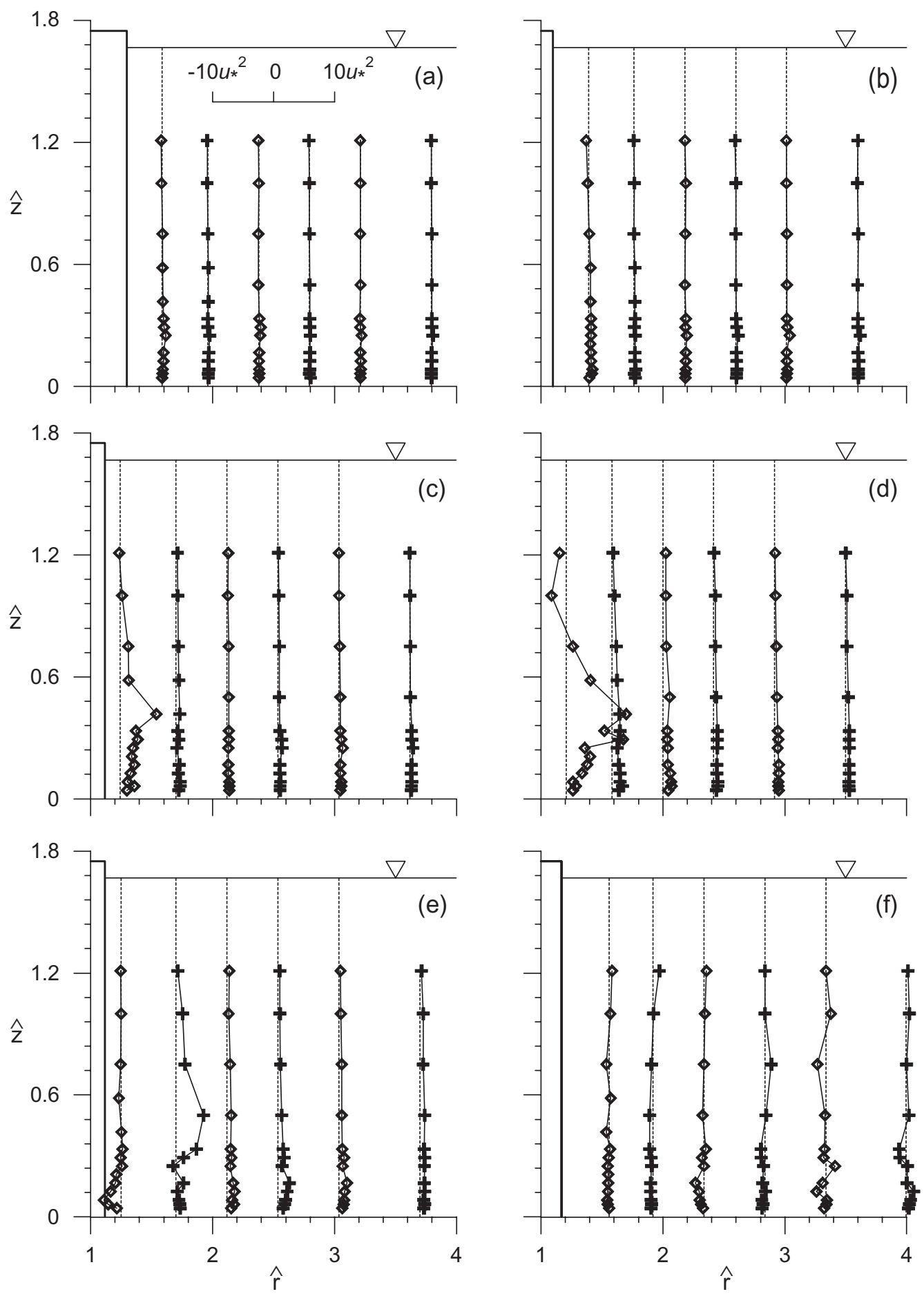

Figure 13. Vertical profiles of $u w^{+}$at azimuthal sections $\theta=10^{\circ}$ (a), $30^{\circ}(\mathbf{b}), 63.4^{\circ}(\mathbf{c}), 90^{\circ}$ (d), $116.6^{\circ}(\mathbf{e})$ and $170^{\circ}$ (f). 

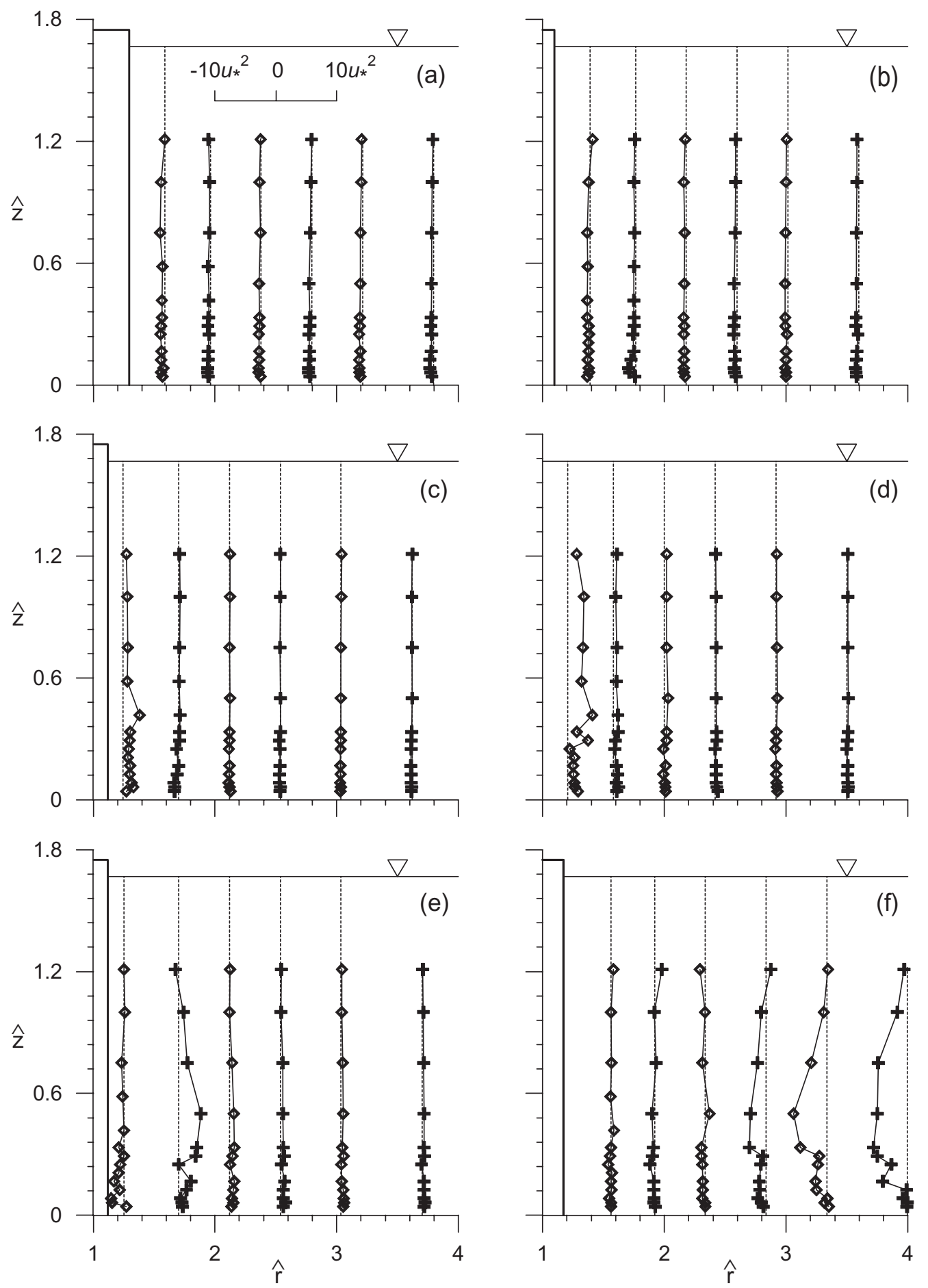

Figure 14. Vertical profiles of $v w^{+}$at azimuthal sections $\theta=10^{\circ}(\mathbf{a}), 30^{\circ}(\mathbf{b}), 63.4^{\circ}(\mathbf{c}), 90^{\circ}(\mathbf{d})$, $116 \cdot 6^{\circ}(\mathbf{e})$ and $170^{\circ}$ (f). 
Velocity and turbulence at a wing-wall abutment
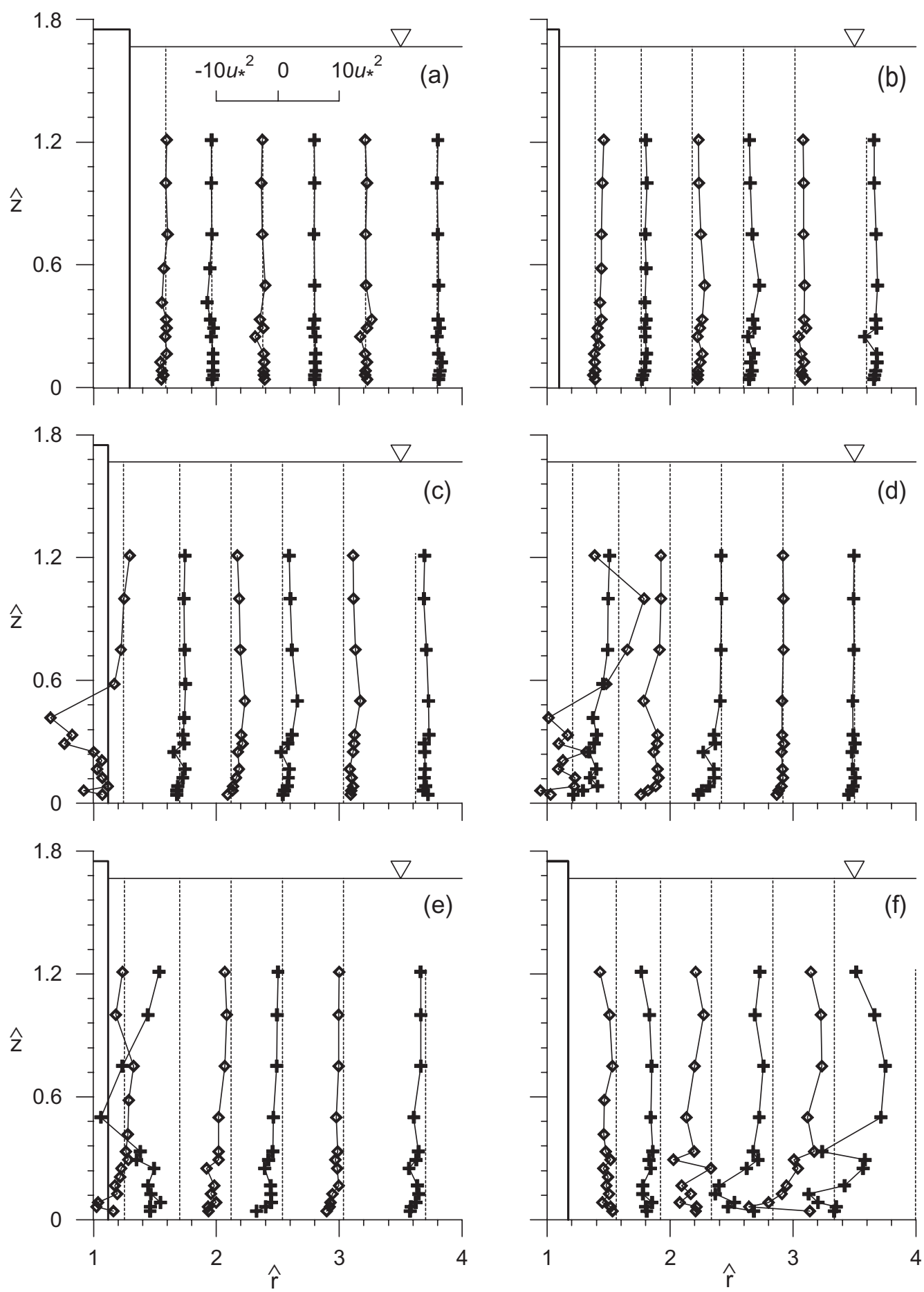

Figure 15. Vertical profiles of $u v^{+}$at azimuthal sections $\theta=10^{\circ}(\mathbf{a}), 30^{\circ}(\mathbf{b}), 63.4^{\circ}(\mathbf{c}), 90^{\circ}(\mathbf{d})$, $116 \cdot 6^{\circ}(\mathbf{e})$ and $170^{\circ}(\mathbf{f})$. 
Table 1. Values of eddy viscosity for different azimuthal angles.

\begin{tabular}{lcccccc}
\hline$\theta(\mathrm{deg})$ & 10 & 30 & 63.4 & 90 & 116.6 & 170 \\
\hline$\varepsilon\left(\mathrm{m}^{2} / \mathrm{s}\right)$ & $8.15 \times 10^{-6}$ & $9.68 \times 10^{-6}$ & $1.16 \times 10^{-5}$ & $2 \cdot 19 \times 10^{-5}$ & $2.25 \times 10^{-5}$ & $2 \cdot 38 \times 10^{-4}$ \\
\hline
\end{tabular}
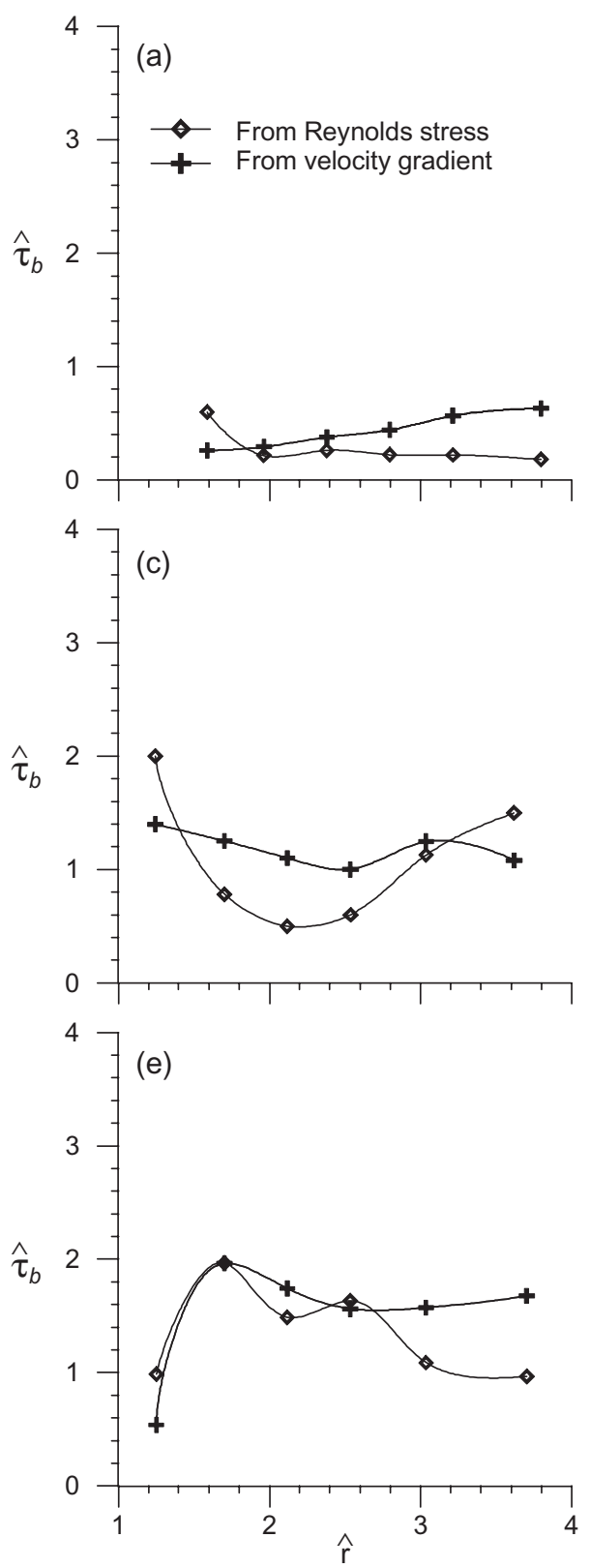

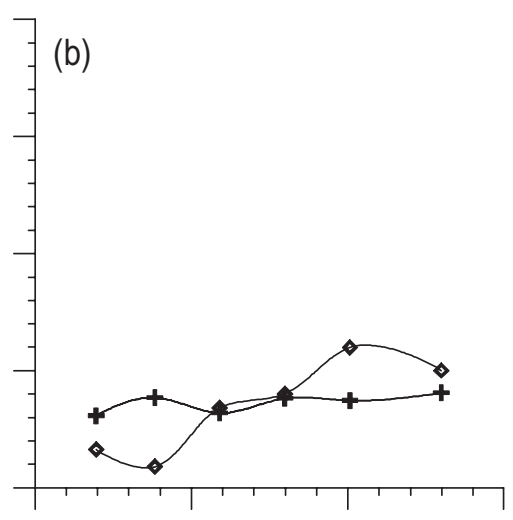

(d)
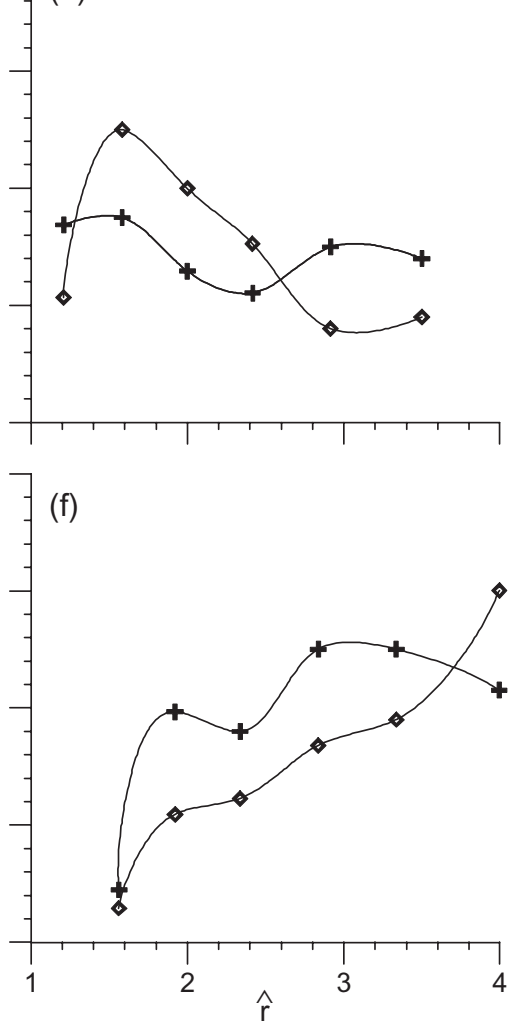

Figure 16. Variations of $\hat{\tau}_{b}$ with $\hat{r}$ at azimuthal sections $\theta=10^{\circ}$ (a), $30^{\circ}$ (b), 63.4 $(\mathbf{c}), 90^{\circ}$ (d), $116 \cdot 6^{\circ}(\mathbf{e})$ and $170^{\circ}$ (f). 
The results of bed shear stress, shown in figure 16, being sufficient to develop a scour hold (if the abutment were embedded in loose sediment beds), can be used to estimate the size of riprap stones or boulders to be used to prevent the formation of scour holes at the base of the abutments.

\section{Conclusions}

The measurement of 3D turbulent flow field around a $45^{\circ}$ wing-wall abutment was made in a laboratory flume using an Acoustic Doppler Velocimeter (ADV). The profiles of timeaveraged velocity components, turbulent intensity components, turbulent kinetic energy and Reynolds stresses at different azimuthal planes have been presented. In the upstream, it is evident from the flow vectors at azimuthal and horizontal planes that a primary vortex associated with the downflow exists. On the other hand, in the downstream, the flow is reversed associated with strong shedding of wake vortex and high Reynolds stresses. The bed shear stresses have been determined from the Reynolds stresses and velocity gradients.

\section{List of symbols}

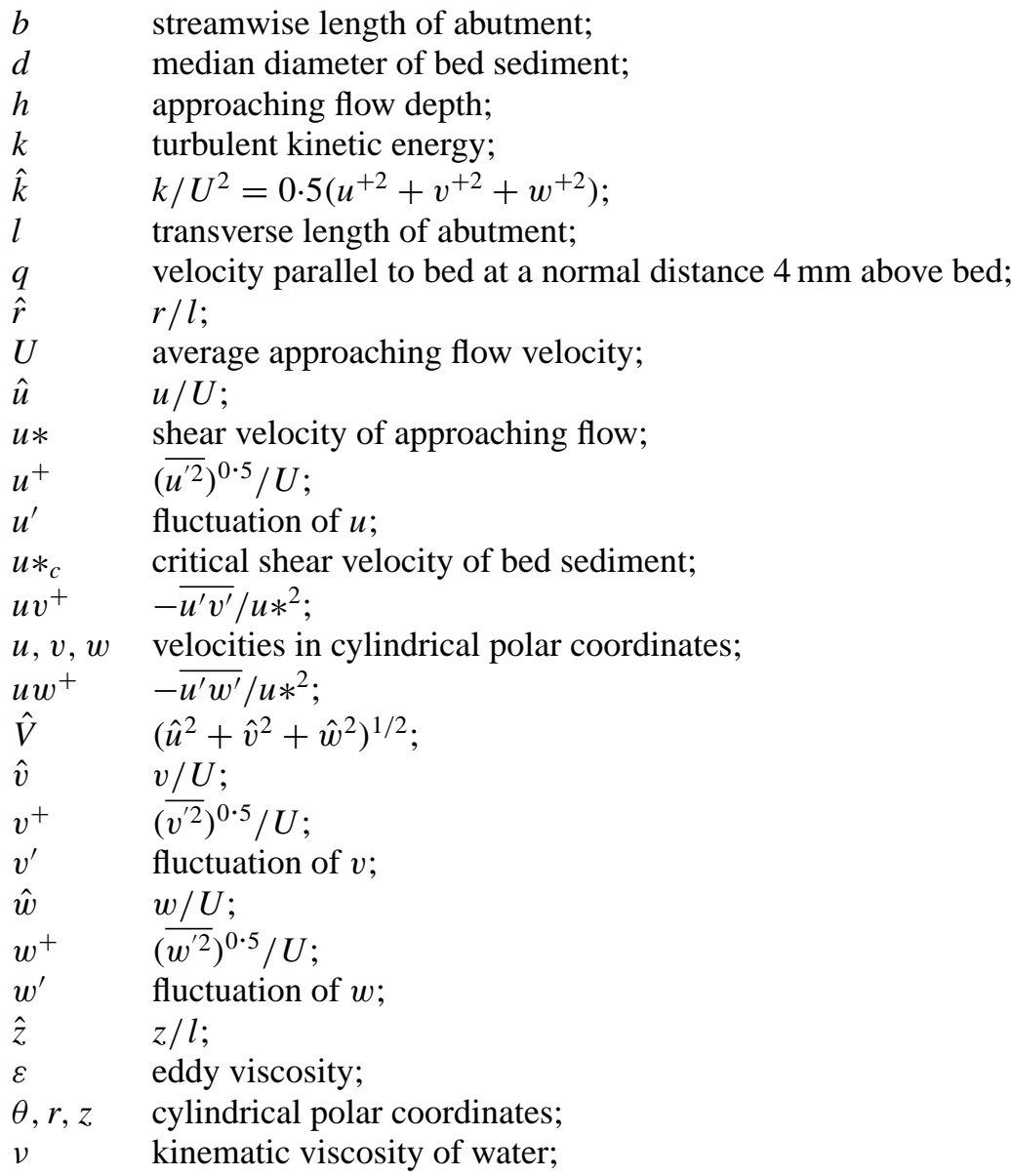


$\rho \quad$ mass density of water;

$\sigma_{g}$ geometric standard deviation;

$\tau_{o} \quad$ bed shear stress of approaching flow;

$\tau_{b} \quad$ local bed shear stress;

$\hat{\tau}_{r} \quad \tau_{b} / \tau_{o}$

$\tau_{r} \quad$ radial bed shear stress;

$\tau_{\theta} \quad$ tangential bed shear stress.

\section{References}

Ahmed F, Rajaratnam N 2000 Observations on flow around bridge abutment. J. Hydraul. Eng. Am. Soc. Civil Eng. 126: 51-59

Barbhuiya A K 2003 Clear water scour at abutments. Ph D thesis, Department of Civil Engineering, Indian Institute of Technology, Kharagpur, India

Dey S 1995 Three-dimensional vortex flow field around a circular cylinder in a quasi-equilibrium scour hole. Sādhanā 20: 771-785

Dey S, Bose S K, Sastry G L N 1995 Clear water scour at circular piers: a model. J. Hydraul. Eng. Am. Soc. Civil Eng. 121: 869-876

Graf W H, Yulistiyanto B 1998 Experiments on flow around a cylinder: The velocity and velocity fields. J. Hydraul. Res. 36: 637-655

Kwan T F 1989 A study of abutment scour. Rep. No. 451, School of Engineering, University of Auckland, Auckland, New Zealand

Kwan T F, Melville B W 1994 Local scour and flow measurements at bridge abutments. J. Hydraul. Res. 32: 661-673

Melville B W, Raudkivi R J 1977 Flow characteristics in local scour at bridge piers. J. Hydraul. Res. 15: $373-380$

Odgaard A J 1986 Free-surface air core vortex. J. Hydraul. Eng. Am. Soc. Civil Eng. 112: 610-620

Rajaratnam N, Nwachukwu B A 1983 Flow near groyne-like structures. J. Hydraul. Eng. Am. Soc. Civil Eng. 109: 463-480

Rozovksii IL 1961 Flow of water in bends of open channels. Israel Program for Scientific Transactions, Jerusalem, Israel 